\title{
DNA Extraction from Pulp Tissue for its Application in PCR. A Study to Extract DNA with High Purity as Quantified by Gel Electrophoresis
}

\author{
Syed Vaseemuddin \\ Reader, Department of Oral Medicine and Radiology, KLES Dental College, Bengaluru, Karnataka, India
}

\begin{abstract}
Correspondence: Syed Vaseemuddin, Reader, Department of Oral Medicine and Radiology, No 157, 4th Cross, Lower Palace
\end{abstract} Orchards, Bengaluru-560003, Karnataka, India, Phone: 98441-74665, e-mail: vaseembds@yahoo.com

\section{ABSTRACT}

The isolation of human genomic DNA is an important step involved in genetic identity analysis. Also getting optimal quantity and quality of DNA is important as this can alter the results. This consideration is especially important in the field of forensic analysis and genetic identity, where DNA is extracted from extremely small amounts of starting material often recovered from suboptimal storage conditions. Odontological examinations have always been a critical determinant in the search of human identity. Teeth form a natural barrier to exogenous DNA contamination. DNA extraction from dental tissues both hard tissues and dental pulp, has been reported the use of dental pulp, as a source of DNA offers a new paradigm in the process of DNA extraction and its use in forensic analysis. ${ }^{11}$ The following procedures elucidate a novel method of extraction of DNA using dental pulp, which offers a cost-effective and attractive procedure, which can be replicated and applied in DNA amplification (PCR).

Keywords: Gel electrophoresis, PCR, Forensics.

\section{INTRODUCTION}

Many molecular biological techniques and investigations require genomic DNA as a starting material, which is especially true for forensic disciplines where the rapid and robust isolation of small quantities of DNA from human tissues is critical. The isolation of human genomic DNA is important step involved in genetic identity analysis. ${ }^{1}$ Many techniques currently used for genomic DNA isolation lead to significant dilutions of the sample material or require the precipitation of genomic material making the isolation of nucleic acids from small amounts of material especially difficult. Also getting optimal quantity and quality of DNA is important as this can alter the results. This consideration is especially important in the field of forensic analysis and genetic identity where DNA is extracted from extremely small amounts of starting material often recovered from suboptimal storage conditions. Odontological examinations have always been a critical determinant in the search of human identity. ${ }^{2}$ Teeth form a natural barrier to exogenous DNA contamination. Teeth are the tissue of choice in the evaluation of DNA sequences when the whole body is destroyed.

In the presenting times, the ubiquitous availability of mass dental care in conjunction with maintenance of dental records has served to enhance the specificity of dental examination as a tool in identification. ${ }^{3}$ Human dentition outlasts any other tissue in the human body and dental tissues serve as an important resource material in forensic investigations, conventionally literature has reported that teeth, dental tissues have always been considered as highly individualistic determinants in identity establishment. ${ }^{4-6}$ In their review of practice of forensic odontology, the applications include not only to routinely encountered identification of solitary deceased but is specially pertinent to the prospect of inevitable mass disasters of increasing seriousness and size involving large populations. Because of the resistant nature of dental tissues to environmental assaults, such as incineration, mutilation dental tissues offer an excellent source in forensic analysis. ${ }^{7,8}$

The most common role of a forensic odontologist is the identification of individuals. Earlier various morphological features like crown size, root lengths have been used to identify an individual when skeletal or mortal remains present insufficient evidence. ${ }^{9,6}$ In tune with technological breakthroughs, newer technologies like DNA evidence, PCR procedures are being amalgamated into the purview of forensic odontology. Thus, the ultimate goal of forensic analysis is to obtain a positive identification from biological evidence samples. ${ }^{10}$

DNA extraction from dental tissues both hard tissues and dental pulp has been reported the use of dental pulp, as a source of DNA offers a new paradigm in the process of DNA extraction and its use in forensic analysis. ${ }^{11}$ The following procedures elucidate a novel method of extraction of DNA using dental pulp, which offers a cost-effective and attractive procedure, which can be replicated and applied in DNA amplification (PCR). 


\section{Objectives}

1. Simple cost-effective protocol for DNA isolation and demonstrate utility of pulp as a source of DNA.

2. Obtain DNA of high degree of purity.

3. Reproducible, simple, and standardized protocol.

\section{Methodology}

The teeth utilized in this study were collected from the department of oral and maxillofacial surgery. They were stored in saline immediately after extraction.

Tooth was cleaned using running tap water. Before preparation, the tooth was treated with $80 \%$ alcohol for five minutes to remove surface decontamination. The pulp chamber was reached from the occlusal surface using a high-speed air rotor hand piece (30,000 rpm). New burs were utilized for each tooth to reduce the risk of contamination. The powder formed during access opening is removed by a 3 way syringe. Pulp was extirpated using a suitable barbed broach in order to avoid shredding of pulp tissues.

The tissue samples thus obtained were stored in individualized vials with saline at $-20^{\circ}$ celsius in a refrigeration compartment, which is then transferred to a laboratory for further tests.

DNA isolation was performed according to the standardized protocol $^{12}$ with a few modifications to obtain DNA devoid of contaminants and high purity yield.

\section{Prepared Solutions}

1. Buffered phenol: Prepared by adding 8-hydroxyquinolone to redistilled liquefied phenol and $50 \mathrm{mMol}$ of tris base of ph 10.5 and $50 \mathrm{mMol}$ tris chloride of ph 8 and TE buffer (Tris, a common $\mathrm{pH}$ buffer, and EDTA, a molecule chelating cations like Mg. ${ }^{2+}$ The purpose of TE buffer is to protect DNA or RNA from degradation.) $\mathrm{pH}$ of 8.

2. Phenol, chloroform and isomyl alcohol mixture (PCI mixture) PCI mixture has a corresponding ratio of phenol, chloroform and isomyl alcohol at 25:24:1 ml of PCI mixture was prepared by adding $25 \mathrm{ml}$ of phenol to $24 \mathrm{ml}$ of chloroform and $1 \mathrm{ml}$ of isomyl alcohol.

3. $1 \times$ SSC (saline-sodium citrate) buffer: $1 \mathrm{ml}$ of $20 \times$ SSC buffer was diluted with $20 \mathrm{ml}$ of triple distilled, nuclease free water to prepare $20 \mathrm{ml}$ of $1 \times$ SSC buffer.

\section{Stages of DNA Isolation}

1. Pulp tissue was ground using a prechilled mortar and pestle, which was maintained at a temperature of -20 degree centigrade before being used. The pulp was ground and 500 mic L of TE buffer was added. The buffer served as a media for transporting the ground pulp.

2. The contents in the mortar were transferred into a microfuge tube using Eppendorf micropipettes of $500 \mathrm{mic}$ l capacity.
3. 250 mic L of TE buffer was added to the mortar using a micropipette and the contents in the mortar mixed well and this was transferred into the same tube and the tube capped firmly. This step helped in transfer of the remnant tissue and to ensure complete transfer of the sample.

4. $1 \mathrm{ml}$ of SSC buffer was added and centrifugation was done for 2 minutes at $12000 \mathrm{rpm}$ in a refrigerated micro centrifuge to separate the components. The result of centrifugation was the formation of a supernatant and a pellet of tissue at the bottom of microfuge tube.

5. Following the centrifugation, the supernatant formed was discarded using an Eppendorf micropipette with disposable tips. In order to avoid contamination 375 mic $\mathrm{L}$ of $0.2 \mathrm{M}$ sodium acetate was added to the vial and vortexing was done in order to dissolve the formed pellet completely.

6. To this 25 mic L of SDS solution (sodium lauryl sulfate) and 5 mic L of proteinase $\mathrm{K}$ soln $(20 \mathrm{mg} / \mathrm{ml})$, which helps in exclusion of protein contaminants by protein digestion, were added vortex briefly and incubate for 2 hours at $55^{\circ} \mathrm{C}$.

7. 120 mic L of PCI (phenol, chloroform, isomyl alcohol) was added to the microfuge tube vortexed for 30 seconds centrifuge for 2 minutes at 12000 rpm, phenolic extraction of DNA was done using the PCI mixture following centrifugation an aqueous supernatant containing DNA and a viscous layer consisting mainly of phenol and chloroform is formed at the bottom of tube.

8. The aqueous layer was removed carefully with an Eppendorf micropipette to avoid phenol inclusion and transferred to a new $1.5 \mathrm{ml}$ microfuge tube.

9. The above two steps were repeated and $1 \mathrm{ml}$ of $100 \%$ cold ethanol was added to remove the residues formed.

10. The contents gently agitated and incubated for 30 minutes at $-20^{\circ} \mathrm{C}$.

11. Centrifugation done at $4^{\circ} \mathrm{C}$ for 5 minutes at $12000 \mathrm{rpm}$.

12. The supernatant thus formed was decanted and drained to avoid any ethanol residues.

13. 186.4 mic L of 10:1 TE Buffer was added and vortexed and incubated for 10 minutes.

14. 14.13.6 mic $\mathrm{L}$ of $3 \mathrm{M}$ sodium acetate was added and $100 \%$ cold ethanol was added. Centrifugation was done at $4^{\circ} \mathrm{C}$ at $12000 \mathrm{rpm}$ to wash away residual contaminants.

15. The supernatant was decanted and the pellet was rinsed with $1 \mathrm{ml}$ of $80 \%$ ethanol centrifuged yet again at $12000 \mathrm{rpm}$.

16. The nucleic acids are found as a pellet or grains on the side of the tube, the supernatant was decanted and the pellet was dried by inverting the tube on a blotting paper.

17. The pellet was resuspended in $100 \mathrm{mic} \mathrm{L}$ of TE buffer.

18. Overnight incubation at $55^{\circ} \mathrm{C}$ for priming of the DNA for storage and the resultant DNA is stored at a temperature of $-20^{\circ} \mathrm{C}$ in a refrigerated compartment. After the extraction of the nucleic acids (DNA) Electrophoresis is done in order to check the positive finding of presence of DNA on an 
agarose gel, this is known as agarose gel electrophoresis and was done as follows.

\section{AGAROSE GEL ELECTROPHORESIS}

Electrophoresis is the movement of particles in an electrical field towards one or the other electric poles. Agarose is a purified form of agar gel used in the electrophoretic studies for the separation of charged molecules and serves as a medium for movement of DNA to the opposite pole on application of an electric current.

DNA is a negatively charged molecule and moves by electric current through the matrix of agarose towards the positively charged anode.

\section{AGAROSE GEL PREPARATION}

Materials: $1 \times$ TAE (TAE buffer is a buffer solution containing a mixture of Tris base, acetic acid and EDTA. Buffer, agarose $0.8 \%$ gel.

$1 \times$ TAE buffer is prepared by diluting stock solution of TAE buffer, which is at a concentration of $50 \times$, by diluting $49 \mathrm{ml}$ of triple distilled water and $1 \mathrm{ml}$ of $50 \times$ TAE buffer.

\section{Gel Preparation}

$0.4 \mathrm{gm}$ of agarose was added to $50 \mathrm{ml}$ of $1 \times$ TAE buffer and heated on an electric mantle till agarose completely melts. This was cooled to the room temperature and ethidium bromide, which is a nucleic acid dye was added, the mixture was then poured into the gel casting trays made of UV transparent plastic. The sample combs used to make wells in the gel are placed gently into the casting tray before the agarose solidified. After the gel was solidified, the combs were removed taking care not to rip off the bottom of the wells.

\section{SAMPLE LOADING}

The gel tray was flooded with electrophoresis buffer TAE, and the samples containing 20 mic 1 of DNA and $5 \mathrm{ML}$ Bromopthalein dye, which acts as a 'Dye front', were pipetted into the sample wells formed by the combs. The lid and power leads were placed on the electrophoresis apparatus and an electric current of 55 voltsis applied for a maximum of 2 hours or till a visible movement of the dye front was seen migrating at least half the length of gel.

Ethidium bromide is used to stain nucleic acid and incorporated into agarose gels prior to gel pouring. Nucleic acid stained with ethidium bromide can be visualized under UV light.

On obtaining a positive finding of DNA, the DNA was quantified using a UV spectrophotometer to check the quantity of DNA and its purity in each case for its use in polymerase chain reaction based amplification.

\section{UV QUANTIFICATION}

The procedure was performed using $200 \mathrm{ml}$ silica cuvettes, into which 20 mic 1 of DNA sample was added and the rest of the volume, i.e $180 \mathrm{ml}$ was made up by adding TE buffer. Along with each sample, a cuvette with $200 \mathrm{mic}$ l of TE buffer was used as a 'blank' or control, which gives an optical density of 0 , hence served to distinguish and assess the samples.

The cuvettes were placed in the Beckman DU 65 spectrophotometer and optical density was read at 260 and 280 nanometers.

The DNA concentration was calculated by measuring the optical density atv 260 multiplied by dilution factor and 50 the samples showed an average concentration of about 60-70 $\operatorname{mic}$ gm/l

The purity was calculated by calculating the optical density at 260 to the optical density at 280, a ratio of 1.7:2 was obtained, thus excluding protinaceous or RNA contamination.

\section{DISCUSSION}

Forensic applications are being recognized in a greater number today as it has been widely accepted to be valuable evidence in medico legal identification in mass disasters. DNA analysis and identifying an individual from the remains caused a great revolution in the field of forensics. ${ }^{13,14}$ DNA as a material for amplification procedures in recognition of gender, age and other determinants of identity is now being routinely performed.

Around $20 \%$ of the whole human DNA consists of a sequence of nucleic acids, which are very likely to repeat. This is also called as a satellite DNA. The human genome contains at least several predominant satellite DNA sequences. The number of copies of a satellite DNA varies over a wide range so that remarkable comparisons can be identified that discriminate between two persons. This biological discovery has led to a strategy and technology sufficient to discriminate between siblings and between siblings and their parents. ${ }^{14}$

One of the perplexing factors encountered has been the standardization and optimization of evidence material when the available tissue samples are contaminated or very less. ${ }^{1}$ In a previous study, which utilized DNA samples of tooth and soft tissue, it was shown that DNA samples extracted from tooth were easily amplified with PCR as DNA samples from tissues contained a PCR inhibitor, which was removed on further dilution.

The above study was performed to arrive at a reproducible, reliable and cost-effective procedure of DNA isolation. DNA extraction from dental tissues has been reported in literature. A considerable amount of research has also been done on the effect of storage conditions and period on the end results. ${ }^{11,15-17}$ Our study shows that normal saline serves as an excellent source of storage media.

The pulp tissue was subjected to phenolic extraction though commonly used is fraught with short comings as the phenol and chloroform, SDS solutions are potent PCR inhibitors, two modifications were done to the standard procedure during the course of isolation:

1. Centrifugation and vortexing cycles were extended for homogenization.

2. Subjected to longer centrifugation after addition of cold ethanol and 3M sodium acetate. 
These steps resulted in obtaining DNA of good quality as observed on the UV spectrophotometer. The study coludes with opinion of authors, who have reviewed extensively on dental pulp as a DNA source. The average DNA quantity ranged in 60 to $70 \mathrm{mcg}$, which is consistent with previous reported methods.

The presenting study then fulfilled the primary objectives of isolating DNA from dental pulp tissues and to have a costeffective and reproducible method.

\section{REFERENCES}

1. Kephart B. Rapid isolation of genomic DNA from small quantities of human tissue. Geneprint 1999;7-9.

2. Pretty IA, Sweet D. A look at forensic dentistry. BDJ14 2001; 90(7):359-66.

3. Wilson DF, Brown KA. Forensic odontology. 1997;55: 752-53.

4. Lessig R, Edellman J. Individualization of dental tissue: An aid for odontological identification. J for Odontostomatology 1995;1:1-3.

5. Michall Bowers, lain Pretty. Arguments on the individuality of human teeth: From the presentation at the 2000 American Academy of Forensic Science.

6. Sweet D, DiZinnio JA. Personal identification through dental evidence: From tooth fragments to DNA. J Calif Dent Assc 1996;24(5):35-42.
7. Sarangi M, Maini M, Sharma G. The practice of forensic odontology. JIDA 1995;66:120-23.

8. Pretty A, David Sweet-www.forensicdentistryonline.org/ forensicpages1/identguide.htm. 2004.

9. Sweet DJ, Sweet CHW. 'DNA analysis of dental pulp to link incinerated remains of homicide victim to the crime' J For Sci 1995;40(2):310-14.

10. Cecilia von Beroldingen, Edward Blake T, Russell Higuchi. Applications of PCR to analysis of biological evidence. PCR technology 209-25.

11. Hai Slavkin. Sex, Enamel and Forensic dentistry: A search for identity. JADA Jul 1997;128(7):1021-25.

12. DNA extraction from tissues. Protocol-9. Manuale Genomix'99. 30-32

13. Nesser D. Leicht-Galati sex determination of forensic samples by simultaneous PCR amplification of alphoid satelloid family DNA from both X and Y Chromosomes. J For Sci Mar 1995;40(2).

14. Preseda Z, Brkie H. Acta Stomatol Croat 2000;21-24.

15. Christian Ruithberg M, Dennis Reeder J, John Butler M. STR Base: Short tandem repeat DNA database for human identity testing'. Nucleic Acid Research 2001;29(1):320-22.

16. DNA Forensics. Oak Ridge: Oak Ridge National Library/Human Genome Project 2001(20).

17. Nabeel Affara, Malcolm Fergusson. DNA sequence homology between sex chromosomes, molecular genetics of sex determination 1994;225-57. Academic Press Inc. 